\title{
Some structural aspects of neodymium praseodymium oxalate single crystals grown in hydro silica gels
}

\author{
CYRIAC JOSEPH, M A ITTYACHEN and K S RAJU* \\ School of Pure and Applied Physics, Mahatma Gandhi University, Kottayam 686031, India \\ *Department of Crystallography and Biophysics, University of Madras, Guindy Campus, \\ Madras 600025 , India
}

MS received 25 September 1996; revised 14 November 1996

\begin{abstract}
The mixed crystals of neodymium praseodymium oxalate are grown by the diffusion of a mixture of aqueous solutions of neodymium nitrate and praseodymium nitrate (as an upper reactant) into the set gel embedded with oxalic acid. By varying the concentration (by volume) of rare earth nitrates in the upper reactant, the incorporation of $\mathrm{Nd}$ and $\mathrm{Pr}$ in the mixed crystals has been studied. Tabular crystals with the well defined hexagonal basal planes are observed in the mixed crystals of varying concentrations. X-ray diffraction patterns of these powdered samples reveal that these mixed crystals are 'isostructural', while IR and FTIR spectra establish the presence of oxalate groups. TGA and DSC analyses show the correctness of the chemical formula for the mixed crystals, by the release of water molecules (endothermic) and of $\mathrm{CO}$ and $\mathrm{CO}_{2}$ (exothermic), with the rare earth oxides as the stable residue. X-ray fluorescence (XRF) and energy dispersive X-ray analyses (EDAX) establish the presence of heavy rare earth elements qualitatively and to a good extent quantitatively. X-ray photoelectron spectroscopic (XPS) studies confirm the presence of rare earth elements (Nd and $\mathrm{Pr}$ ) as their respective oxides. The findings of these techniques of characterization are in excellent agreement with the proposed empirical structure for the mixed rare earth oxalates. The implications are discussed.
\end{abstract}

Keywords. Neodymium praseodymium oxalate crystals; growth; XRD; IR; FTIR; TGA; XRF; EDAX; XPS.

\section{Introduction}

The interesting physical properties of rare earths have drawn the attention of material scientists (Eyring 1964) and a variety of materials containing rare earths have gained recognition for their magnetic and luminescent properties (Kotru and Raina 1986; Galczynski and Strek 1991; Zhang Yugeng et al 1993). The ionic conductivity of rare earth oxalates $\left[\mathrm{R}_{2}\left(\mathrm{C}_{2} \mathrm{O}_{4}\right)_{3} \cdot n \mathrm{H}_{2} \mathrm{O}\right](\mathrm{R}=\mathrm{Nd}, \mathrm{Pr}, \mathrm{Sm}, .$.$) has initiated much$ interest besides its easy conversion into the corresponding oxides (Mohan Rao and Jagannatha 1991).

Since oxalates, molybdates and tartrates are sparingly soluble in water and decompose before melting, high temperature crystal growth methods are not suitable. The growth of crystals in hydro silica gels at room temperature (Henisch 1970) has been fully exploited in the case of mixed rare earth oxalates (Raju et al 1994), molybdates (Sushma Bhat et al 1995; Jayakumari Isac et al 1996) and tartrates (Raju et al 1996), besides their characterization employing several techniques. The growth of mixed neodymium praseodymium oxalate (NPO) crystals in silica gels and their characterization employing X-ray diffraction, optical and infrared absorption spectroscopic methods have been recently reported (Cyriac Joseph et al 1995). From the X-ray

*Author for correspondence 
diffraction studies of powdered neodymium, praseodymium and their mixed oxalates, they have shown that these samples are crystalline and isostructural, belonging to monoclinic system of unit cell parameters: $a=11 \cdot 228, b=9 \cdot 630, c=10.298 \AA$ and $\beta=114 \cdot 28^{\circ}$, matching quite well with each other.

In the present paper, NPO crystals are grown in hydro silica gels by changing the concentration (by volume) of the upper reactants and characterized employing X-ray diffraction (XRD), IR and FTIR, TGA, XRF, EDAX and XPS techniques. These results throw light on the kinetics of chemical reaction, the thermal stability, qualitative and quantitative analyses of heavy elements in the mixed rare earth oxalates besides their chemical states, which in turn, help in proposing the empirical structure of NPO crystals.

\section{Growth of NPO with varying concentrations}

In order to grow mixed oxalates of $\left(\mathrm{Nd}_{x} \operatorname{Pr}_{1-x}\right), 1 \mathrm{M}$ oxalic acid is added to sodium meta silicate solution (specific gravity: $1.03 \mathrm{~g} \cdot \mathrm{cm}^{-3}$ ), which acts as the lower reactant and also adjusts the $\mathrm{pH}$ of the gel to 7 , in the present case.

For growing the crystals of varying concentrations of rare earths in $\left(\mathrm{Nd}_{x} \operatorname{Pr}_{1-x}\right)$ oxalates, the upper reactant is prepared as follows. In the mixture of aqueous solutions of $1 \mathrm{M}$ neodymium nitrate and $1 \mathrm{M}$ praseodymium nitrate $(100 \mathrm{ml})$, the amounts of neodymium nitrate (by volume) are gradually increased in steps of $20,40,50,60$ and $80 \mathrm{ml}(x=0.2,0.4,0.5,0.6$ and 0.8$)$, and the remaining being praseodymium nitrate, thereby making the total volume $100 \mathrm{ml}$ in all the cases.

The above gel with oxalic acid is allowed to set in a test tube $(15 \mathrm{~mm}$ dia, $150 \mathrm{~mm}$ in length) and after setting, the upper reactants of varying concentrations are slowly added over the gel surface, so as to harvest NPO crystals, having varying quantities of $\mathrm{Nd}$ and $\mathrm{Pr}$. The mixed crystals of $\mathrm{Nd}_{x} \mathrm{Pr}_{1-x}\left(\mathrm{C}_{2} \mathrm{O}_{4}\right)_{3} \cdot n \mathrm{H}_{2} \mathrm{O}$ are formed by the diffusion of the upper reactant into the set gel having $1 \mathrm{M}$ oxalic acid (lower reactant) according to the following reaction

$$
\left.\begin{array}{c}
\mathrm{Nd}_{x}\left(\mathrm{NO}_{3}\right)_{3} \\
+ \\
\operatorname{Pr}_{1-x}\left(\mathrm{NO}_{3}\right)_{3}
\end{array}\right]+3 \mathrm{H}_{2} \mathrm{C}_{2} \mathrm{O}_{4} \rightarrow \mathrm{Nd}_{x} \operatorname{Pr}_{1-x}\left(\mathrm{C}_{2} \mathrm{O}_{4}\right)_{3} \cdot n \mathrm{H}_{2} \mathrm{O}+6 \mathrm{HNO}_{3}
$$

The proposed empirical structure for $\mathrm{Nd}_{x} \operatorname{Pr}_{1-x}\left(\mathrm{C}_{2} \mathrm{O}_{4}\right)_{3}$ is given in figure 1 .

Tabular crystals, having well defined edges and corners, with hexagonal basal planes (figure 2) are observed in the mixed NPO samples of varying concentrations. It may be mentioned that the morphology of the mixed crystals is the same and as the concentration (by volume) of neodymium nitrate in the upper reactant increases, the bluish pink coloration predominantly increases in the brownish background.

\subsection{Characterization of NPO}

2.1a XRD studies: The XRD patterns of powdered NPO $(x=0.2,0.5$ and 0.8$)$ samples (figure 3 ) are recorded in a Philips XRD PW 1050/70 with $\mathrm{Cu} \mathrm{K}_{\alpha}$ radiation $(\lambda=1.5418 \AA)$. Well defined Bragg peaks at specific ' $2 \theta$ ' angles in the diffraction patterns suggest that the NPO of varying concentrations are 'ordered', meaning 


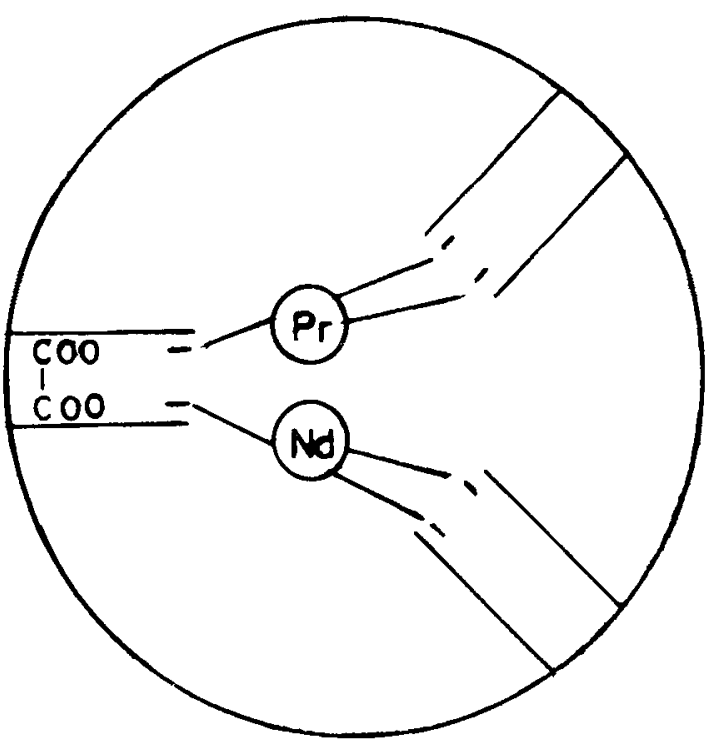

Figure 1. Proposed empirical structure of NPO.

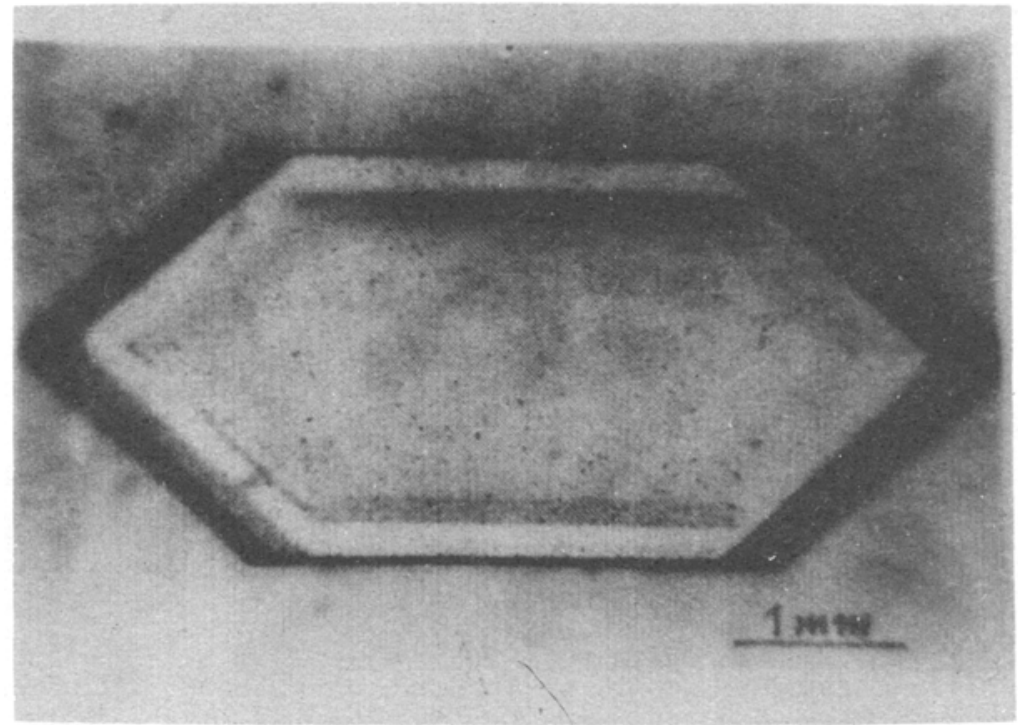

Figure 2. A gel grown crystal of NPO.

crystalline in nature. It is interesting to observe that the ' $2 \theta$ ' values and to some extent the intensities relating to the Bragg peaks show a very good matching in the diffraction patterns for NPO $(x=0 \cdot 2,0.4$ and $0 \cdot 8)$, suggesting that these mixed crystals too, are 'isostructural'.

2.1b IR and FTIR studies: The infrared absorption spectrum of NPO $(x=0 \cdot 2)$ in $\mathrm{KBr}$ matrix is recorded in a Perkin Elmer model 540 (figure 4a). That the sample has water of crystallization is evidenced by the intense peak at $3300 \mathrm{~cm}^{-1}$ relating to 


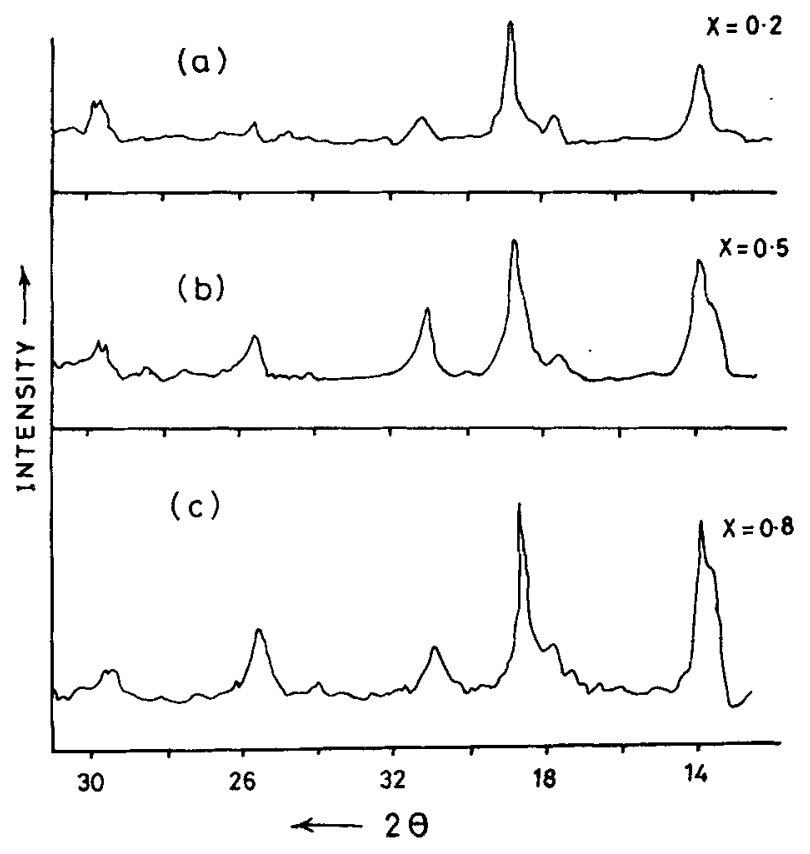

Figure 3. XRD patterns of powdered NPO (a) $x=0 \cdot 2$, (b) $x=0.5$ and (c) $x=0.8$.

asymmetric and symmetric $\mathrm{OH}$ stretchings and the peak at $1630 \mathrm{~cm}^{-1}$ relating to $\mathrm{HOH}$ bending plus $v_{\mathrm{a}}(\mathrm{C}=\mathrm{O})$.

The peaks positioned at $1380 \mathrm{~cm}^{-1}$ and the more intense at $1320 \mathrm{~cm}^{-1}$ are attributed to the symmetric stretchings of $\mathrm{CO}_{2}$. The sharp peak at $810 \mathrm{~cm}^{-1}$ is due to the combined effects of in-plane deformation of $\mathrm{CO}_{2}$ and the presence of metal-oxygen bonds. The medium peak at $480 \mathrm{~cm}^{-1}$ relates to the rocking of $\mathrm{CO}_{2}$.

In order to ascertain the chemical groups of NPO $(x=0 \cdot 8)$ with respect to NPO $(x=0 \cdot 2)$, FTIR of the former, is recorded in $\mathrm{KBr}$ matrix using BRUKER IFS $66 \mathrm{~V}$, FTIR spectrometer (figure 4b).

The peaks relating to the oxalate groups, water of crystallization and metal-oxygen bonds observed for NPO $(x=0 \cdot 2)$ in IR spectrum are in excellent agreement with the FTIR peaks for $\mathrm{NPO}(x=0.8)$ confirming the presence of the identical chemical groups in both the samples (compare figures $4 \mathrm{a}$ and $\mathrm{b}$ ).

2.1c TGA and DSC of NPO: The thermogravimetric and DSC plots of NPO $(x=0 \cdot 5)$ were taken using Delta series TGA by scanning at $20^{\circ} \mathrm{C} / \mathrm{min}$ (figure 5).

The following points merit discussion:

(I) The loss of weight in the temperature range around $100^{\circ} \mathrm{C}$ relates to the loss of water of crystallization (endothermic reaction) as follows.

$$
\mathrm{Nd}_{x} \mathrm{Pr}_{1-x}\left(\mathrm{C}_{2} \mathrm{O}_{4}\right)_{3} \cdot n \mathrm{H}_{2} \mathrm{O} \rightarrow \mathrm{Nd}_{x} \operatorname{Pr}_{1-x}\left(\mathrm{C}_{2} \mathrm{O}_{4}\right)_{3}+n \mathrm{H}_{2} \mathrm{O} \text {. }
$$

(II) The loss of weight around $420^{\circ} \mathrm{C}$ has two chemical changes, one relating to release of $\mathrm{CO}$ at $400^{\circ} \mathrm{C}$ and the other at $430^{\circ} \mathrm{C}$, relating to $\mathrm{CO}_{2}$, which are exothermic in character and very close to each other. The chemical reaction is as follows:

$$
\mathrm{Nd}_{x} \mathrm{Pr}_{1-x}\left(\mathrm{C}_{2} \mathrm{O}_{4}\right)_{3} \rightarrow\left(\mathrm{Nd}_{x} \mathrm{Pr}_{1-x}\right) \mathrm{O}_{3}(s)+3 \mathrm{CO}(g)+3 \mathrm{CO}_{2}(g) \text {. }
$$




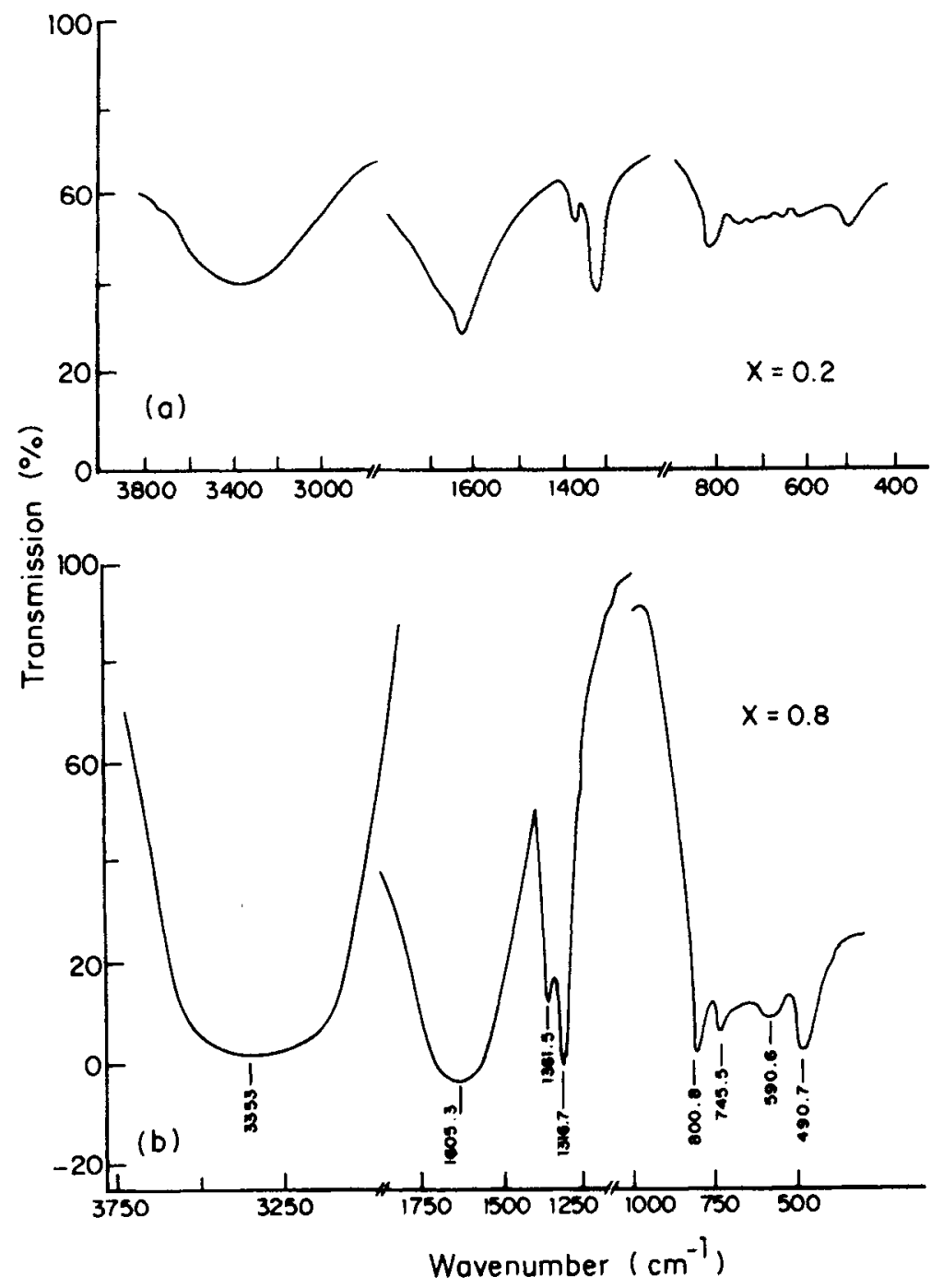

Figure 4. (a) IR absorption spectrum of NPO $(x=0.2)$ and (b) FTIR spectrum of NPO $(x=0 \cdot 8)$.

(III) Beyond $620^{\circ} \mathrm{C}$, the residue mainly consists of $\left(\mathrm{Nd}_{x} \operatorname{Pr}_{1-x}\right) \mathrm{O}_{3}$, which is fairly stable.

These observations are in agreement with the chemical formula for NPO as $\mathrm{Nd}_{x} \operatorname{Pr}_{1-x}\left(\mathrm{C}_{2} \mathrm{O}_{4}\right)_{3} \cdot n \mathrm{H}_{2} \mathrm{O}$.

2.1d XRF of NPO: For subsequent techniques of characterization, NPO is nicely powdered and pellets of $1 \mathrm{~cm}$ diameter and $1 \mathrm{~mm}$ thickness are formed using a hydraulic press by applying a pressure of $1 \cdot 1$ tons per sq.cm. XRF spectra for NPO $(x=0 \cdot 2)$ and $(x=0.8)$ are recorded in Philips X-ray spectrometer model 1410/20 (30 mA, $35 \mathrm{kV})$, pulse height $25 / 30$ with the rhodium $X$-ray tube (counting time of $2 \mathrm{sec}$ ) employing LiF 220 as the X-ray monochromator. 


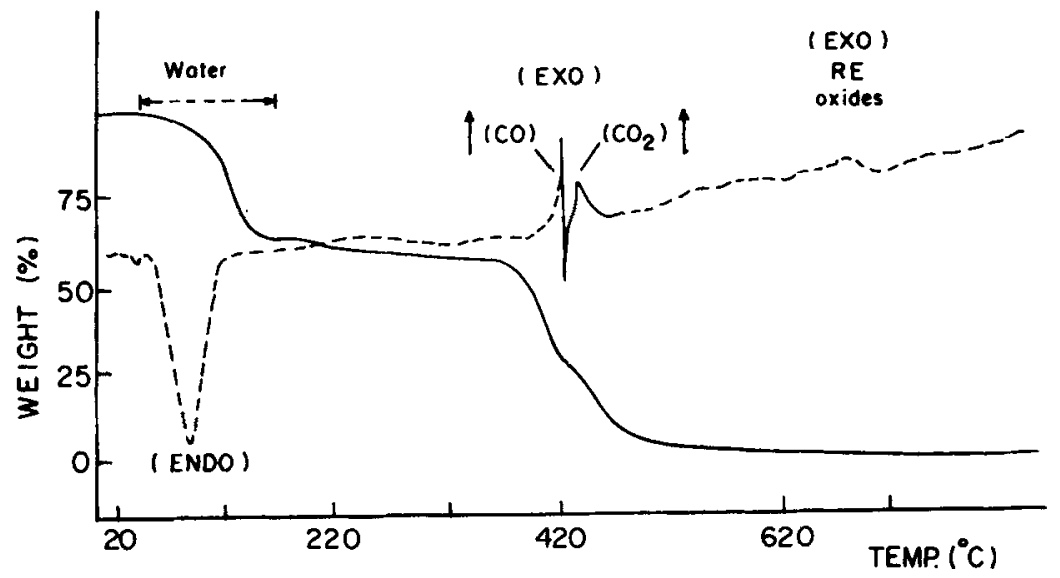

Figure 5. TGA and DSC plots of NPO $(x=0.5)$.

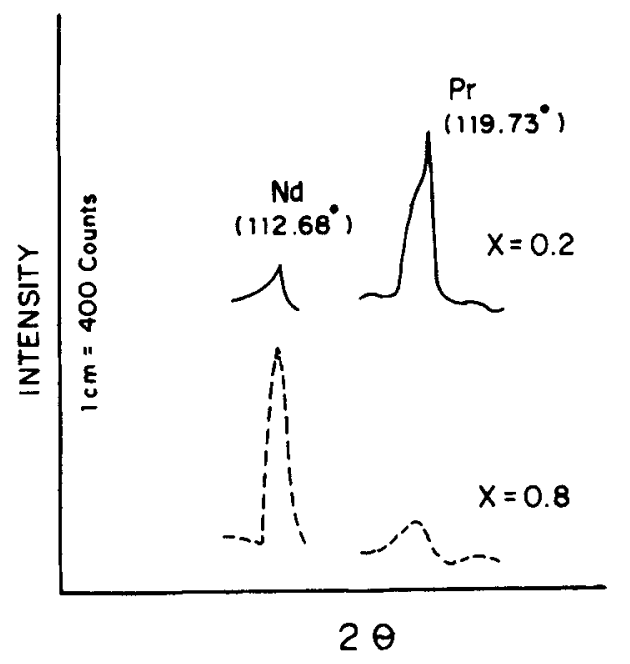

(a)

(b)

Figure 6. XRF spectra of NPO (a) $x=0 \cdot 2$ (continuous lines) and (b) $x=0.8$ (dashed lines).

The XRF spectrum for $\mathrm{NPO}(x=0 \cdot 2)$ is shown as solid lines in figure $6 \mathrm{a}$ and for NPO $(x=0.8)$, as dotted lines in figure $6 \mathrm{~b}$, in which the incorporation of $\mathrm{Nd}$ and $\mathrm{Pr}$ is established by their characteristic $(L \alpha)$ peaks positioned at ' $2 \theta^{\prime}$ ' values $112.68^{\circ}$ and $119 \cdot 73^{\circ}$ respectively in both the samples. Using the ' $d$ ' values relating to $220 \mathrm{LiF}$ monochromater and the equation

$$
n \lambda=2 d \sin \theta,
$$

(Joseph 1960), the ' $2 \theta^{\prime}$ 'values relating to $\mathrm{Nd}\left(112 \cdot 68^{\circ}\right)$ and $\operatorname{Pr}\left(119.73^{\circ}\right)$ give their characteristic wavelengths $(\lambda)$ for the X-ray fluorescent emission lines as 2.370 and $2.643 \AA$, which match exactly with the reported values in the literature (Emmet 1967).

It is indeed interesting to observe that the heights and/or areas for $\mathrm{Nd}(L \alpha)$ and $\operatorname{Pr}$ $(L \alpha)$ peaks for NPO $(x=0 \cdot 2)$ and $(x=0 \cdot 8)$ are directly related to their concentrations (compare figures $6 \mathrm{a}$ and $6 \mathrm{~b}$ ) though not very accurate. This gives a strong clue, that 
XRF technique is a powerful tool to detect heavy elements (Nd and Pr in the present case) in the samples, qualitatively and to some extent quantitatively.

2.1e EDAX studies of NPO: In order to further confirm the incorporation of rare earths in NPO of varying concentrations, EDAX work has been taken up. The pellets of NPO are mounted onto aluminium stubs and examined in an EDAX attachment of SEM (Cambridge stereoscan type 360), after coating the surfaces with a layer of gold, to make them electrically conducting. The EDAX patterns of $\left(\mathrm{Nd}_{x} \operatorname{Pr}_{1-x}\right)$ oxalates for $x=0 \cdot 2,0 \cdot 4,0 \cdot 5,0.6$ and 0.8 are recorded and the typical patterns of EDAX for $x=0 \cdot 2$, 0.5 and 0.8 are shown in figures $7 \mathbf{a}-\mathbf{c}$.

Attention is drawn to the following points which merit discussion.

(I) The two prominent peaks positioned at 5.03 and $5.488 \mathrm{keV}$ (figure $7 \mathrm{a}$ ) correspond quite well to $L_{\alpha}$ and $L_{\beta}$ energies of praseodymium, which are also present in figures $7 \mathrm{~b}$ and $\mathrm{c}$.

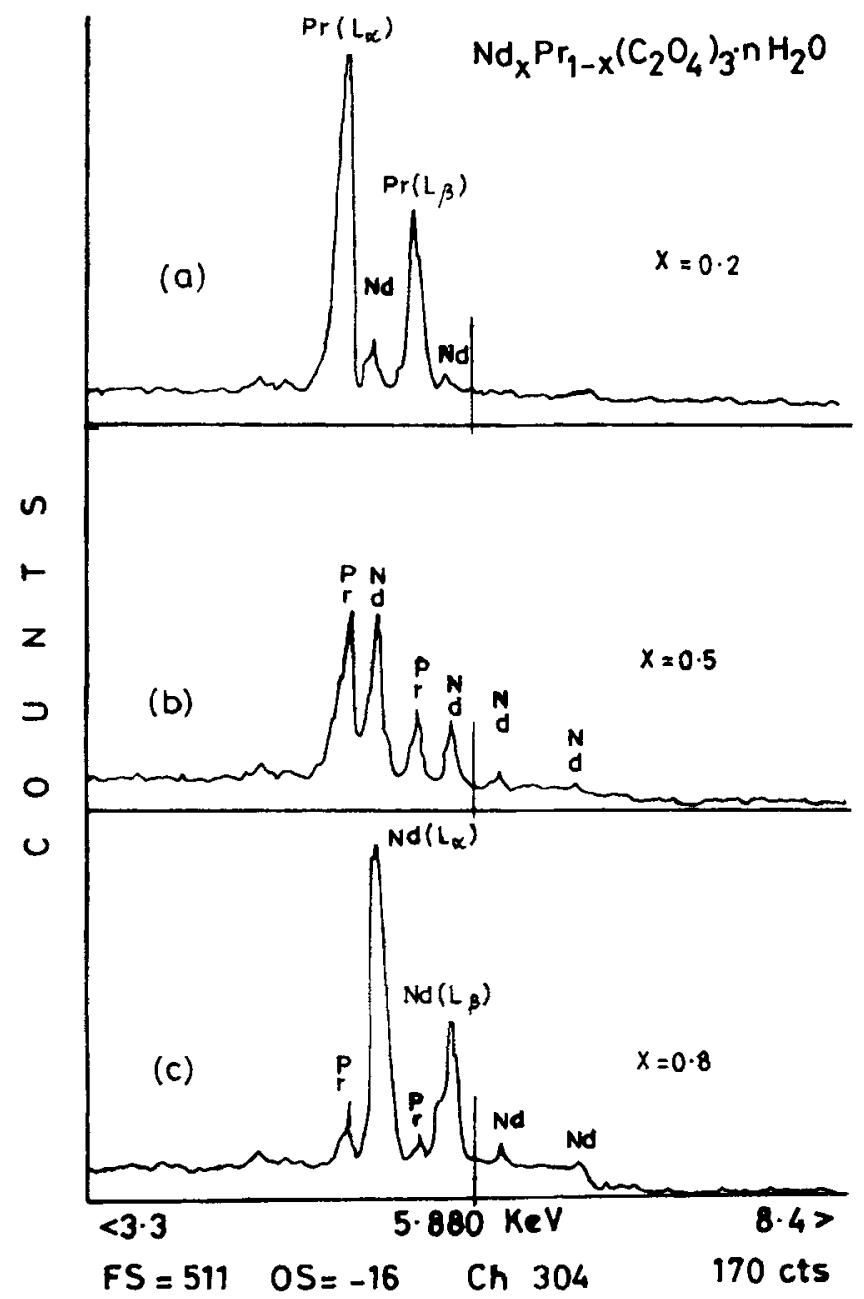

Figure 7. EDAX patterns of NPO (a) $x=0 \cdot 2$, (b) $x=0.5$ and (c) $x=0 \cdot 8$. 
Table 1. Atomic weight percentages of $\mathrm{Nd}$ and $\mathrm{Pr}$ in the mixed crystals of varying concentrations.

\begin{tabular}{lccc}
\hline & $\begin{array}{c}\text { Vol. conc. of the } \\
\text { upper reactant } \\
\text { Expt. }\end{array}$ & $\begin{array}{c}\text { At. weight \% from EDAX } \\
\text { analyses }\end{array}$ \\
\hline 1 & $\mathrm{Nd}\left(\mathrm{NO}_{3}\right)_{3}: \operatorname{Pr}\left(\mathrm{NO}_{3}\right)_{3}$ & $\mathrm{Nd} L_{\alpha}$ & $\operatorname{Pr} L_{\alpha}$ \\
\hline 2 & $20: 80$ & 17.292 & 82.708 \\
3 & $40: 60$ & 37.930 & 62.070 \\
4 & $50: 50$ & 49.169 & 50.831 \\
5 & $60: 40$ & 61.014 & 38.986 \\
\hline
\end{tabular}

(II) The two prominent peaks positioned at 5.23 and $5.72 \mathrm{keV}$ (figure 7c) relate to the $L_{\alpha}$ and $L_{\beta}$ of neodymium, which are obviously present in figures $7 \mathrm{a}$ and $\mathrm{b}$.

(III) The peaks relating to $L_{\alpha}$ of $\mathrm{Nd}$ and $\mathrm{Pr}$ have almost equal heights and areas for $x=0.5$, in NPO crystals (left hand side of figure 7b).

(IV) As the concentration by volume of $\mathrm{Nd}\left(\mathrm{NO}_{3}\right)_{3}$ in the upper reactant goes on increasing, the heights and areas of $L_{\alpha}$ peaks of $\mathrm{Nd}$ also go on increasing (compare in figures 7a, b and c), while the heights and areas of $L_{\alpha}$ peaks of $\operatorname{Pr}$ go on decreasing as the concentration by volume of $\operatorname{Pr}\left(\mathrm{NO}_{3}\right)_{3}$ decreases, and vice versa.

The EDAX data giving the atomic weight percentages of $\operatorname{Nd} L_{\alpha}$ and $\operatorname{Pr} L_{\alpha}$ for varying $x$ in NPO are given in table 1 . It is indeed interesting to note that the atomic weight percentages of $\mathrm{Nd}$ and $\mathrm{Pr}$ from EDAX data have a very good correlation with the concentration by volume (i.e. $x$ values for $\mathrm{Nd}$ and $(1-x)$ for $\mathrm{Pr}$ ).

2.1f XPS studies of NPO $(x=0 \cdot 5)$ : In order to further establish the chemical states of the rare earth constituents incorporated in NPO $(x=0.5)$, XPS work has been taken up. XPS spectra of NPO pellet are recorded in VG scientific ESCA LAB MK II, by irradiating the sample with the monochromatic soft X-rays ( $\mathrm{Mg} \mathrm{K}_{\alpha}$ : Photon energy $1253.6 \mathrm{eV}$ ). The constant analyser energy is $50 \mathrm{eV}$ and the resolution of the system is about $0.7 \mathrm{eV}$.

The XPS peak relating to $\mathrm{Nd}\left(3 d_{5 / 2}\right)$ having the binding energy value at $980 \mathrm{eV}$ is shown in figure 8 , while figure 9 shows the XPS peak relating to $\operatorname{Pr}\left(3 d_{5 / 2}\right)$ with the binding energy centred at $930 \mathrm{eV}$.

The XPS region of carbon $1 s$ of NPO sample(figure 10) shows two peaks having binding energies centred around 287 and $290 \mathrm{eV}$. The first peak positioned at $287 \mathrm{eV}$ is attributed to the adventitious hydrocarbon as a result from the laboratory environment or from the glove box, while the second peak at the higher binding energy value of $290 \mathrm{eV}$ relates to the oxalate $\left(\mathrm{C}_{2} \mathrm{O}_{4}\right)$ group. The second peak relating to the oxalate group is shifted with respect to the first peak towards the higher binding energy value and this is attributed due to higher electronegativity of oxalate group. It is obviously clear that XPS is a powerful tool, for non destructive surface analysis, in not only detecting the individual constituents in the sample, but also their chemical states as well.

\section{Discussion}

That the NPO grown in hydrosilica gels for varying concentrations $(x=0 \cdot 2,0 \cdot 4,0 \cdot 5,0 \cdot 6$ and 0.8$)$ are crystalline, is evidenced by the well defined edges and corners, with the 


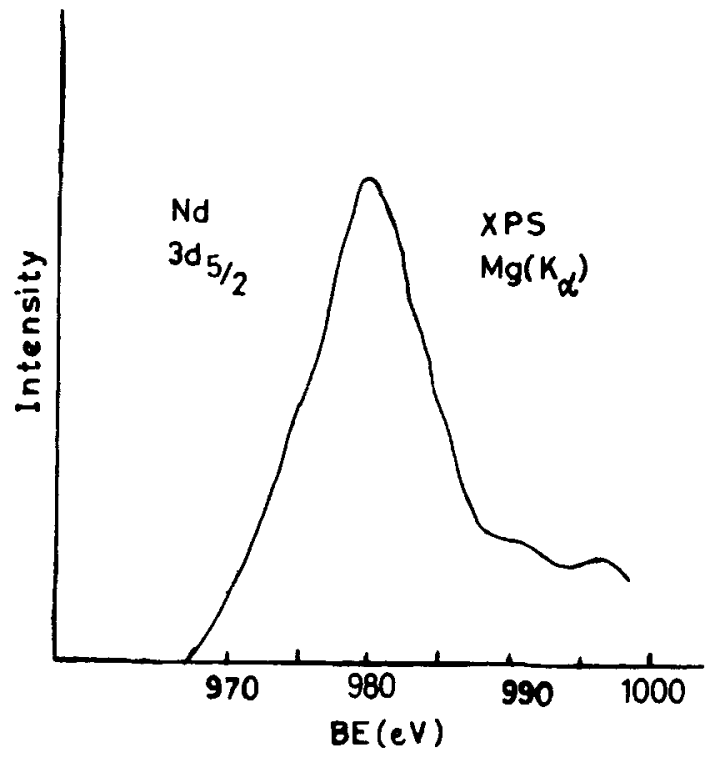

Figure 8. XPS peak relating to $\mathrm{Nd}\left(3 d_{5 / 2}\right)$ in NPO.

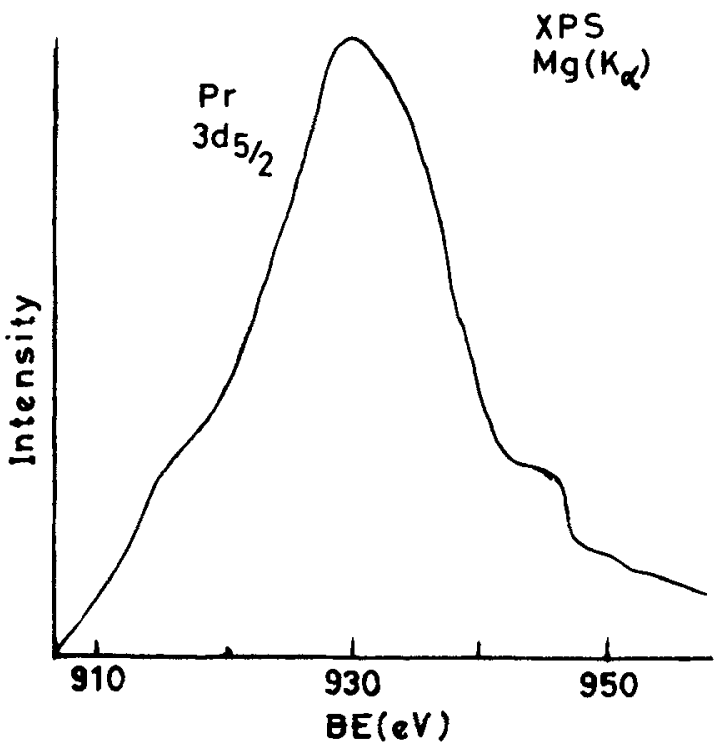

Figure 9. XPS peak relating to $\operatorname{Pr}\left(3 d_{5 / 2}\right)$ in NPO.

hexagonal basal planes (figure 2). The XRD patterns of the above powdered NPO samples reveal well defined Bragg peaks at specific ' $2 \theta$ ' angles meaning that the samples are crystalline in nature (figure 3a) and show identical XRD patterns for NPO (figures $3 a-c)$, with very good matching of the ' $2 \theta$ ' values corresponding to their respective Bragg peaks, suggesting that the above samples are 'isostructural'. According to the earlier report of the authors (Cyriac Joseph et al 1995), the XRD patterns of powdered oxalates of neodymium (NO), praseodymium (PO) and their mixed rare earths (NPO) relate to 'monoclinic system' with the unit cell parameters $a=11 \cdot 228, b=9.630$, 


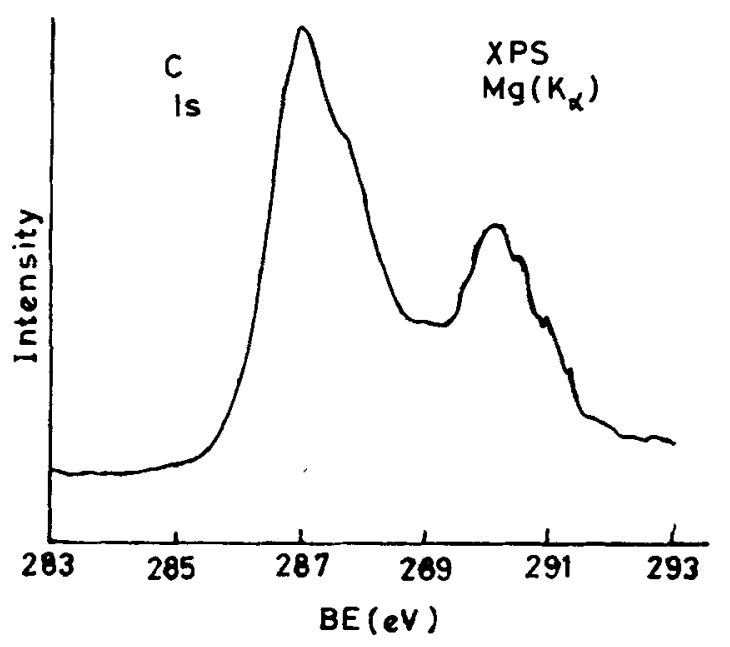

Figure 10. XPS peak of C $1 s$ of NPO.

$c=10 \cdot 298 \AA$ and $\beta=114 \cdot 28^{\circ}$. Since, NPO of varying concentrations $(x)$, grown in the present case, are 'isostructural' (figure 3 ), it is concluded that these mixed crystals of NPO also belong to 'monoclinic system'.

The observed peaks in IR and FTIR of NPO $(x=0 \cdot 2)$ and $(x=0.8)$ respectively, relating to oxalate groups, water of crystallization and metal oxygen bond are in excellent agreement with the general crystal structure for simple metal oxalates (Fujita 1957). The small shifts in $\mathrm{cm}^{-1}$ values of peaks observed in IR and FTIR relating to NPO $(x=0.2)$ and $(x=0.8)$ may be attributed due to the change in the chemical environment caused by the predominance of $\mathrm{Nd}$ over $\mathrm{Pr}$, as the concentration of $\mathrm{Nd}(x)$ is increased during the growth.

The TGA and DSC experiments for NPO support the proposed empirical structure $\left(\mathrm{Nd}_{x} \mathrm{Pr}_{1-x}\right)\left(\mathrm{C}_{2} \mathrm{O}_{4}\right)_{3} \cdot n \mathrm{H}_{2} \mathrm{O}$ (figure 5) by the physical and chemical changes during 'pyrolysis'. The endothermic physical change of the mixed crystals correspond to dehydration around $100^{\circ} \mathrm{C}$ and the exothermic chemical changes around $400^{\circ} \mathrm{C}$ due to the evolution of $\mathrm{CO}$ and $\mathrm{CO}_{2}$, thereby resulting in the stable residue, $\mathrm{Nd} \operatorname{Pr}\left(\mathrm{O}_{3}\right)$ above $620^{\circ} \mathrm{C}$.

XRF studies establish the incorporation of Nd and Pr in NPO for $x=0.2$ and 0.8 in quality and to some extent in quantity, by the revelation of XRF emission peaks at the characteristic ' $2 \theta$ ' values. It is worth mentioning that the wavelengths of these XRF peaks calculated from ' $2 \theta$ ' and ' $d$ ' values of $\mathrm{LiF}(220)$ match quite well with the literature (Emmet 1967).

That the mixed oxalate crystals of $\mathrm{Nd}$ and Pr grown by the diffusion of their nitrates into the set gel embedded with oxalic acid contain neodymium and praseodymium, is obviously evident by the revelation of EDAX peaks relating to $\operatorname{Nd} L_{\alpha, \beta}$ and $\operatorname{Pr} L_{\alpha, \beta}$ in figures $7 \mathrm{a}-\mathrm{c}$ respectively. The energy values $(\mathrm{keV})$ relating to $L_{\alpha, \beta}$ of $\mathrm{Nd}$ and $\operatorname{Pr}$ match exactly with the EDAX library, thereby confirming the presence of $\mathrm{Nd}$ and $\mathrm{Pr}$ in their mixed oxalates.

The atomic weight percentages of $\mathrm{Nd} L_{\alpha}$ and $\operatorname{Pr} L_{\alpha}$ in the mixed rare earth oxalates have reasonable correlation with the concentration by volume of their respective nitrates (table 1). The heights and or areas (meaning the measure of the quantitative contents of their concerned elements (Riggs and Parker 1975) of the $L_{\alpha}$ peaks of Nd and 
$\operatorname{Pr}$ (figures $7 \mathrm{a}-\mathrm{c}$ ) are directly related to their respective nitrate concentrations in the upper reactant. If the concentration by volume $(x)$ of rare earth $(\mathrm{Nd})$ nitrate increases, the heights and areas of the corresponding ' $L_{\alpha}$ peaks' also increase and vice versa.

These results strongly confirm that EDAX, though a surface analysis, is a powerful tool for the detection of heavy elements in the sample, in quality and also in quantity, without the destruction of the sample, unlike in chemical reaction methods.

XPS studies establish the presence of Nd and $\mathrm{Pr}$ in NPO besides their chemical states as their respective oxides $\left(\mathrm{Nd}_{2} \mathrm{O}_{3}\right.$ and $\left.\operatorname{Pr}_{2} \mathrm{O}_{3}\right)$, agreeing quite well with the earlier findings of the authors (Raju et al 1994; Jayakumari et al 1996). These rare earth oxides are formed, as a result of getting linked to $\mathrm{O}^{-}$of $\left(\mathrm{C}_{2} \mathrm{O}_{4}\right)$ groups (figures 8 and 9), while the XPS of C $1 s$ of NPO (figure 10) proves the presence of oxalate group as well. These results are in excellent agreement with the proposed empirical formula for NPO (figure 1).

\section{Conclusions}

From the present work on gel grown NPO of varying concentrations, the following conclusions are drawn.

(i) The crystallinity of NPO sample is evidenced by the well defined edges and corners, with the hexagonal basal planes (figure 2).

(ii) NPO samples are ordered meaning crystalline and are 'isostructural', belonging to monoclinic system (XRD).

(iii) The presence of oxalate complexes accounted by the revelation of characteristic peaks and of lattice water by the broad peak at $3200-3600 \mathrm{~cm}^{-1}$ (IR and FTIR).

(iv) The correctness of the chemical formula for $\mathrm{NPO}$ as $\mathrm{Nd}_{x} \operatorname{Pr}_{1-x}\left(\mathrm{C}_{2} \mathrm{O}_{4}\right)_{3} \cdot n \mathrm{H}_{2} \mathrm{O}$ has been proved by its dehydration around $100^{\circ} \mathrm{C}$ (endothermic) and evolution of $\mathrm{CO}$ and $\mathrm{CO}_{2}$ around $420^{\circ} \mathrm{C}$ (exothermic), yielding stable residual rare earth oxides beyond $600^{\circ} \mathrm{C}$ (TGA and DSC).

(v) The presence of $\mathrm{Nd}$ and $\mathrm{Pr}$ in NPO has been established qualitatively and quantitatively (XRF and EDAX), and

(vi) The NPO has $\mathrm{Nd}$ and $\mathrm{Pr}$ in their oxide states (XPS) getting linked to $\mathrm{O}^{-}$of oxalate groups (figure 1).

\section{Acknowledgements}

One of the authors (KSR) thanks the Chairman, Materials Research Centre, Indian Institute of Science, Bangalore for IR and EDAX and Regional Sophisticated Instrumentation Centre, Indian Institute of Technology, Madras for FTIR, TGA and ESCA facilities.

\section{References}

Cyriac Joseph, George Varghese and Ittyachen M A 1995 Cryst. Res. Technol. 30159

Emmet F K (ed.) 1967 Hand Book of X-rays (New York: McGraw Hill Book Company) 1.14 to 1.18

Eyring L 1964 Progress in science and technology of rare earths (New York: Pergamon Press)

Fujita M 1957 J. Phys. Chem. 671014 
Galczynski M and Strek W 1991 J. Phys. Chem. Solids 52681

Henisch H K 1970 Crystal growth in gels (Pennsylvania: Pennsylvania University Press)

Jayakumari Isac, Ittyachen M A and Raju K S 1996 Bull. Mater. Sci. 19495

Joseph V S (ed.) 1960 Index to $X$-ray Powder Data File (Philadelphia, USA: ASTM) p. 70

Kotru P N and Raina K K 1986 J. Phys. D. Appl. Phys. 19 L9

Mohan Rao P and Jagannatha N 1991 Current trends in crystal growth and characterization (Mysore: M I T Associates Pvt Ltd)

Raju K S, Krishna K N, Jayakumari Isac and Ittyachen M A 1994 Bull. Mater. Sci. 171447

Raju K S, Anima Jain, Sushma Bhat and Kotru P N 1996 Indian J. Pure \& Appl. Phys. 34784

Riggs W M and Parker M J 1975 Methods of surface analysis (ed.) A W Czanderna (Amsterdam: Elsevier) Chap 4, p. 108

Sushma Bhat, Kotru P N and Raju K S 1995 Curr. Sci. 69607

Zhang Yugeng, Su Qinde and Zhao Guiwen 1993 Cryst. Res. \& Technol. 28995 